\title{
DIVERSIDADE DE CARRAPATOS (ACARI: IXODIDAE) EM ANIMAIS SILVESTRES RECEBIDOS PELO ZOOLÓGICO MUNICIPAL DE GUARULHOS
}

\author{
TICK DIVERSITY (ACARI: IXODIDAE) ON WILD ANIMALS RECEIVED BY THE \\ MUNICIPAL ZOO OF GUARULHOS
}

\section{T. F. MARTINS ${ }^{1}$, C. A. IGAYARA-SOUZA ${ }^{2}$, T. C. SANCHES ${ }^{3}$, M. A. MELO ${ }^{4}$, C. E. BOLOCHIO ${ }^{5}$, A. A. NAGAHAMA ${ }^{6}$, H. W. HIDASI ${ }^{7}$, G. N. PENIDO JUNIOR ${ }^{8}$, I. C. L. ACOSTA ${ }^{9}$, S. MUÑOZ-LEAL ${ }^{10}$, M. B. LABRUNA ${ }^{11}$}

\section{RESUMO}

O Zoológico Municipal de Guarulhos, localizado no estado de São Paulo, recebe e recupera animais silvestres do próprio município e de alguns municípios limítrofes. Entre os anos de 1997 e 2016 foram coletados carrapatos de animais silvestres (Cariama cristata, Buteo brachyurus, Alouatta guariba, Bradypus variegatus, Tamandua tetradactyla, Puma concolor, Cerdocyon thous, Nasua nasua, Mazama gouazoubira, Didelphis aurita, Sphiggurus villosus, Myocastor coypus e Hydrochoerus hydrochaeris) atendidos pelo Zoológico. Foram identificadas as seguintes espécies de carrapatos: Amblyomma aureolatum (Pallas, 1772), Amblyomma calcaratum Neumann, 1899, Amblyomma dubitatum Neumann, 1899, Amblyomma longirostre (Koch, 1844), Amblyomma nodosum Neumann, 1899, Amblyomma parkeri Fonseca e Aragão, 1952, Amblyomma sculptum Berlese, 1888, Amblyomma varium Koch, 1844, Haemaphysalis juxtakochi Cooley, 1946, Ixodes loricatus Neumann, 1899 e Rhipicephalus sanguineus sensu lato (Latreille, 1806). Apesar de todas as associações carrapato-hospedeiro já terem sido previamente relatadas no território brasileiro, o presente estudo registra pela primeira vez um ginandromorfo de A. dubitatum.

PALAVRAS-CHAVE: Amblyomma. Ginandromorfismo. Haemaphysalis. Ixodes. Rhipicephalus.

\section{SUMMARY}

The Municipal Zoo of Guarulhos, located in the state of São Paulo, receives and recovers wild animals from the Guarulhos municipality and from some neighboring municipalities. Between the years of 1997 and 2016 ticks were collected on wild animals (Cariama cristata, Buteo brachyurus, Alouatta guariba, Bradypus variegatus, Tamandua tetradactyla, Puma concolor, Cerdocyon thous, Nasua nasua, Mazama gouazoubira, Didelphis aurita, Sphiggurus villosus, Myocastor coypus, and Hydrochoerus hydrochaeris) attended by the Zoo. The following tick species have been identified: Amblyomma aureolatum (Pallas, 1772), Amblyomma calcaratum Neumann, 1899, Amblyomma dubitatum Neumann, 1899, Amblyomma longirostre (Koch, 1844), Amblyomma nodosum Neumann, 1899, Amblyomma parkeri Fonseca and Aragão, 1952, Amblyomma sculptum Berlese, 1888, Amblyomma varium Koch, 1844, Haemaphysalis juxtakochi Cooley, 1946, Ixodes loricatus Neumann, 1899, and Rhipicephalus sanguineus sensu lato (Latreille, 1806). Although all tick-host associations have previously been reported in Brazilian territory, the present study records for the first time a gynandromorph of A. dubitatum.

KEY-WORDS: Amblyomma. Gynandromorphism. Haemaphysalis. Ixodes. Rhipicephalus.

\footnotetext{
${ }^{1}$ Faculdade de Medicina Veterinária e Zootecnia, Universidade de São Paulo - São Paulo, SP

${ }^{2}$ Zoológico Municipal de Guarulhos - Guarulhos, SP.

${ }^{3}$ Zoológico Municipal de Guarulhos - Guarulhos, SP.

${ }^{4}$ Zoológico Municipal de Guarulhos - Guarulhos, SP.

${ }^{5}$ Zoológico Municipal de Guarulhos - Guarulhos, SP.

${ }^{6}$ Zoológico Municipal de Guarulhos - Guarulhos, SP.

${ }^{7}$ Zoológico Municipal de Guarulhos - Guarulhos, SP.

${ }^{8}$ Zoológico Municipal de Guarulhos - Guarulhos, SP.

${ }^{9}$ Faculdade de Medicina Veterinária e Zootecnia, Universidade de São Paulo - São Paulo, SP.

${ }^{10}$ Faculdade de Medicina Veterinária e Zootecnia, Universidade de São Paulo - São Paulo, SP.

${ }^{11}$ Faculdade de Medicina Veterinária e Zootecnia, Universidade de São Paulo - São Paulo, SP.

Autor para correspondência. E-mail: thiagodogo@hotmail.com
} 


\section{INTRODUÇÃO}

Os carrapatos são ectoparasitas obrigatórios de extrema importância epidemiológica devido a sua ação deletéria sobre a saúde animal e humana. Danos diretos são causados por hematofagia além da inoculação de agentes infecciosos, desta forma os carrapatos são vetores de um número impressionante de agentes patogênicos (GUGLIELMONE et al., 2003). A domesticação dos animais para fins econômicos ou de companhia favoreceu o estudo de aproximadamente $20 \%$ das espécies de carrapatos, entretanto, pouco se sabe a respeito de $80 \%$ das espécies de carrapatos que parasitam a fauna silvestre (BARROS-BATTESTI et al., 2006). Os Jardins Zoológicos apresentam um conjunto único da interação entre os seres humanos, animais silvestres e artrópodes de importância médicoveterinária, incluindo os carrapatos, que são vetores de patógenos para os animais cativos e humanos. Programas para o controle de ectoparasitas em Zoológicos são comuns, sendo assim, os Parques Zoológicos possuem um papel fundamental no monitoramento e biossegurança, recebendo animais silvestres da natureza que servem como sentinelas para detectar artrópodes exóticos e introdução de doenças transmitidas por vetores. A colaboração entre os acarologistas e os técnicos de Zoológicos ajuda a melhorar a investigação e a saúde pública, protegendo desta forma o público visitante através da educação ambiental e a higidez dos animais em cativeiro. (ADLER et al., 2011).

O Zoológico Municipal de Guarulhos está localizado no estado de São Paulo. Guarulhos é o segundo município mais populoso do estado, sendo a cidade não capital mais populosa do Brasil (IBGE, 2016). Entretanto o município vive um drama diário e silencioso, quase imperceptível, pois a cada dia a ocupação desordenada pelo homem traz uma nova tragédia para os animais silvestres, a diminuição dos seus ambientes naturais e o contato com o ser humano, que pode ser fatal. Animais silvestres estão aparecendo feridos com maior frequência nos últimos anos devido ao avanço da cidade sobre as matas que são o habitat de muitas espécies ameaçadas de extinção (ALMEIDA \& VASCONCELLOS, 2007; PIEDADE, 2013). Por outro lado, uma equipe de veterinários e biólogos especializados no atendimento de animais silvestres trabalha diariamente com a pesquisa e conservação no Zoológico, contra as adversidades, com o objetivo de salvar animais em perigo e reintegrá-los a natureza.

$\mathrm{O}$ presente trabalho objetivou identificar $\mathrm{e}$ relatar a diversidade de carrapatos em animais silvestres recebidos pelo Zoológico Municipal de Guarulhos.

\section{MATERIAL E MÉTODOS}

Entre os anos de 1997 e 2016 foram coletados carrapatos de animais silvestres recebidos pelo Zoológico Municipal de Guarulhos e os mesmos preservados em álcool $70 \%$. Os carrapatos foram identificados em estereomicroscópio, utilizando-se chaves taxonômicas (BARROS-BATTESTI et al., 2006; MARTINS et al., 2010; MARTINS et al., 2016) e posteriormente tombados no acervo da "Coleção Nacional de Carrapatos Danilo Gonçalves Saraiva" (CNC) da Faculdade de Medicina Veterinária e Zootecnia da Universidade de São Paulo, sob os números de tombo (CNC-3023-3035, 3403-3408).

\section{RESULTADOS E DISCUSSÃO}

As associações carrapato-hospedeiro encontradas no presente estudo, com seus respectivos municípios, estão apresentadas na (Tabela 1). No presente estudo foi identificada uma ninfa de Amblyomma sculptum em seriema (Cariama cristata) no município de Mairiporã. Ninfas desta mesma espécie de carrapato também já foram anteriormente identificadas no Brasil em seriemas nos municípios de Jundiaí e Sorocaba, ambas no estado de São Paulo, além do município de Morrinhos localizado no estado de Goiás (LABRUNA et al., 2007; MARTINS et al., 2015; LUZ et al., 2016). Ainda no presente estudo, foram identificadas ninfas desta mesma espécie de carrapato em outra ave, um gavião de cauda curta (Buteo brachyurus), proveniente também do município de Mairiporã. Ninfas do carrapato A. sculptum já foram previamente identificadas no estado de São Paulo em gavião de cauda curta proveniente do município de Rio Grande da Serra (MARTINS et al., 2017). O presente encontro de ninfas de $A$. sculptum em $C$. cristata e $B$. brachyurus no município de Mairiporã corrobora os trabalhos anteriores. A presente identificação de fêmea de A. sculptum em bugio ruivo (Alouatta guariba) no município de Arujá está de acordo com o trabalho recente de Martins et al. (2016), que também identificaram fêmea desta espécie de carrapato em bugio ruivo no município de São Paulo. Ainda no município de Arujá, o presente estudo identificou adultos de Amblyomma varium em duas preguiças comum (Bradypus variegatus), corroborando assim com o trabalho de Martins et al. (2015), que identificaram também adultos do "carrapato gigante da preguiça" em duas B. variegatus provenientes dos municípios de Carapicuíba e Sorocaba.

No município de Bertioga foram encontradas as espécies Amblyomma calcaratum e Amblyomma nodosum parasitando tamanduá mirim (Tamandua tetradactyla), sendo que estas duas espécies de carrapatos foram recentemente encontradas por Martins et al. (2015), parasitando diversos tamanduás mirim de alguns municípios do estado de São Paulo. Na presente pesquisa, neste mesmo hospedeiro foram encontrados um macho e uma ninfa de Rhipicephalus sanguineus sensu lato, sendo que esta mesma espécie de carrapato foi também recentemente encontrada parasitando um tamanduá mirim no Nordeste do Brasil (OLIVEIRA et al., 2017). 
Tabela 1 - Carrapatos coletados em animais silvestres recebidos pelo Zoológico Municipal de Guarulhos, estado de São Paulo, Brasil, entre os anos de 1997 e 2016.

\begin{tabular}{|c|c|c|c|c|}
\hline $\begin{array}{l}\text { Hospedeiros } \\
\text { (número de animais) }\end{array}$ & Municípios & $\begin{array}{l}\text { Datas } \\
\text { (mês-ano) }\end{array}$ & Carrapatos & $\begin{array}{l}\text { Números de tombo } \\
\text { da CNC }\end{array}$ \\
\hline \multicolumn{5}{|l|}{ AVES } \\
\hline Cariama cristata $(1)$ & Mairiporã & $4-2010$ & 1N Amblyomma sculptum & 3023 \\
\hline Buteo brachyurus (1) & Mairiporã & $10-2016$ & $2 \mathrm{~N}$ A. sculptum & 3407 \\
\hline \multicolumn{5}{|l|}{ MAMÍFEROS } \\
\hline Alouatta guariba (1) & Arujá & 3-2007 & 1F A. sculptum & 3024 \\
\hline Bradypus variegatus (2) & Arujá & $8-2003,5-2004$ & $17 \mathrm{M}, 1 \mathrm{~F}$ Amblyomma varium & 3025 \\
\hline Tamandua tetradactyla $(1)$ & Bertioga & $4-2015$ & $\begin{array}{l}\text { 4M, 3F Amblyomma calcaratum; 9M, 3F Amblyomma nodosum; } \\
1 \mathrm{M}, 1 \mathrm{~N} \text { Rhipicephalus sanguineus sensu lato }\end{array}$ & 3408 \\
\hline Puma concolor $(1)$ & São Paulo & $10-2011$ & 23M, 10F Amblyomma aureolatum; $1 \mathrm{M}$ R. sanguineus $\mathrm{s} .1$. & 3028 \\
\hline P. concolor $(1)$ & Arujá & 4-2015 & $1 \mathrm{M}$ A. aureolatum & 3403 \\
\hline Cerdocyon thous (2) & Guarulhos & 4-1997, 9-2012 & 6M, 6F A. aureolatum & 3027 \\
\hline Nasua Nasua (1) & Guarulhos & 4-2016 & 76L Amblyomma sp. & 3404 \\
\hline Mazama gouazoubira (1) & Guarulhos & $10-2012$ & 3M Haemaphysalis juxtakochi & 3029 \\
\hline Didelphis aurita (5) & Guarulhos & $2-2010,10-2012,10-2015$ & 1N Amblyomma dubitatum; 2N A. sculptum; 4M, 7F Ixodes loricatus & $3026,3405,3406$ \\
\hline Sphiggurus villosus (2) & Mairiporã & 3-2001, 9-2012 & $5 \mathrm{M}, 2 \mathrm{~F}$ Amblyomma longirostre & 3031 \\
\hline S. villosus (1) & Arujá & 3-2007 & $2 \mathrm{M}$ A. longirostre & 3032 \\
\hline S. villosus (2) & Guarulhos & 2-2009, 4-2012 & 9M A. longirostre; 3L Amblyomma sp. & 3033 \\
\hline S. villosus (2) & São Paulo & $8-2012,12-2012$ & $2 \mathrm{M}, 1 \mathrm{~F}$ A. longirostre $; 2 \mathrm{M}, 2 \mathrm{~F}$ Amblyomma parkeri & 3034 \\
\hline Myocastor coypus (1) & Guarulhos & $1-2003$ & $29 \mathrm{~N}$ A. dubitatum & 3030 \\
\hline Hydrochoerus hydrochaeris (9) & Guarulhos & $\begin{array}{l}2-2000,3-2003,11-2011,10- \\
2012,8-2013\end{array}$ & $\begin{array}{l}\text { 351M, 73F, 103N, } 1 \mathrm{G} \text { A. dubitatum; 17M, 5F A. sculptum; } \\
\text { 27L Amblyomma sp. }\end{array}$ & 3035 \\
\hline
\end{tabular}

L: larva; N: ninfa; M: macho; F: fêmea; G: ginandromorfo

CNC: Coleção Nacional de Carrapatos "Danilo Gonçalves Saraiva" da Faculdade de Medicina Veterinária e Zootecnia da Universidade de São Paulo. 
Adultos de Amblyomma aureolatum foram encontrados no presente estudo em duas espécies de carnívoros, onça parda (Puma concolor) e cachorro do mato (Cerdocyon thous). Segundo Labruna et al. (2005), está espécie de carrapato é frequentemente encontrada parasitando carnívoros no Brasil durante o estágio adulto, sendo que os mesmos autores também encontraram adultos de A. aureolatum em onça parda e cachorro do mato. Na presente pesquisa ainda foi encontrado um macho de $R$. sanguineus s. 1 . em $P$. concolor. Sánchez-Cordero \& Medellín (2005) encontraram o "carrapato vermelho do cão" em onça parda, entretanto segundo Labruna et al. (2005) essa relação tem maior influência da manutenção em cativeiro de animais silvestres, sendo que provavelmente as presentes infestações (T. tetradactyla e $P$. concolor) ocorreram em cativeiro e não em vida livre. Foram ainda encontradas no presente estudo larvas de Amblyomma sp. em quati (Nasua nasua), estando de acordo desta forma com Labruna et al. (2005) e Witter et al. (2016), que também encontraram larvas de Amblyomma sp. nesta espécie de carnívoro.

A presente identificação de machos de Haemaphysalis juxtakochi em veado catingueiro (Mazama gouazoubira) proveniente do município de Guarulhos está de acordo com os trabalhos prévios de Martins et al. (2015) e Teixeira et al. (2017), que também encontraram esta espécie de carrapato em veados catingueiro recebidos e atendidos pelo Zoológico de Sorocaba. Em cinco gambás de orelha preta (Didelphis aurita), também provenientes do município de Guarulhos, foram identificadas ninfas de Amblyomma dubitatum e A. sculptum, além de adultos de Ixodes loricatus. A identificação destas três espécies de carrapatos na presente pesquisa, corrobora com Ogrzewalska et al. (2012) que também identificaram ninfas de A. dubitatum e A. sculptum (publicado como Amblyomma cajennense), assim como adultos de $I$. loricatus em gambás de orelha preta na região metropolitana de São Paulo. No presente estudo, adultos de Amblyomma longirostre e Amblyomma parkeri foram encontrados parasitando sete ouriços cacheiro (Sphiggurus villosus) provenientes de quatro municípios do estado de São Paulo (Tabela 1). Segundo Labruna et al. (2009), estas espécies de carrapatos durante o estágio adulto são comumente encontradas parasitando ouriços cacheiro no território brasileiro, sendo que os mesmos autores também encontraram adultos de $A$. parkeri em $S$. villosus. Martins et al. (2015) encontraram adultos de A. longirostre em doze $S$. villosus, todos provenientes do município de Sorocaba e Gonzales et al. (2017) encontraram adultos de A. longirostre e A. parkeri em nove $S$. villosus, todos provenientes do município de São Paulo, sendo assim, a presente pesquisa está de acordo com estes três estudos prévios.

Ninfas de A. dubitatum foram identificadas em ratão do banhado (Myocastor coypus) no município de Guarulhos no presente estudo. Labruna et al. (2004) e Teixeira et al. (2017) também registraram esta mesma espécie de carrapato em ratões do banhado provenientes dos municípios de Jundiaí e Sorocaba, respectivamente. A presente identificação desta espécie de carrapato em $M$. coypus confirma que ninfas de A. dubitatum parasitam com frequência esta espécie hospedeira. Em nove capivaras (Hydrochoerus hydrochaeris), também provenientes do município de Guarulhos e encaminhadas ao Zoológico Municipal, foram identificadas as espécies A. dubitatum e A. sculptum, além de larvas de Amblyomma sp. Martins et al. (2015) e Teixeira et al. (2017) também relataram estas espécies de carrapatos em capivaras encaminhadas ao Zoológico de Sorocaba. Por outro lado a presente pesquisa encontrou um ginandromorfo de $A$. dubitatum parasitando uma $H$. hydrochaeris no município de Guarulhos.

Ginandromorfos são indivíduos quiméricos constituídos por tecidos geneticamente masculinos e femininos, os quais expressam fenótipos sexuais condizentes com seu sexo genético, levando ao desenvolvimento de um indivíduo com partes de ambos os sexos (LABRUNA et al., 2002). O ginandromorfismo ocorre na natureza em diversas espécies de vertebrados e invertebrados, incluindo os carrapatos (LABRUNA et al., 2000). O exemplar ginandromorfo de A. dubitatum, apresentou na vista dorsal características masculinas do lado esquerdo e femininas do lado direito, sendo o inverso na vista ventral (Figura 1 A-C). Desta forma, dorsalmente $\mathrm{o}$ lado esquerdo apresentou escudo completo com a ornamentação de macho (escudo castanho claro com uma mancha esbranquiçada longitudinal na porção lateral), com ausência de área porosa na base do capítulo e o lado oposto apresentou escudo dorsal incompleto com a ornamentação típica de fêmea, com a presença de uma área porosa na base dorsal do capítulo. Ventralmente o lado direito apresentou espinhos nas coxas I e IV de macho (espinhos fortes na coxa I e espinho longo na coxa IV) e festões com prolongamentos quitinosos moderados. Já o lado oposto, apresentou espinhos nas coxas I e IV de fêmea (espinhos curtos na coxa I e espinho curto na coxa IV) e festões sem prolongamentos quitinosos. Dois casos de ginandromorfismo de $R$. sanguineus s. 1. já foram relatados no Brasil (PEREIRA \& CASTRO 1945; LABRUNA et al., 2002). Fonseca, 1935 e Labruna et al. (2002), também publicaram dois casos em A. sculptum (publicados como A. cajennense) e Labruna et al. (2000) um caso em Amblyomma oblongoguttatum no território nacional. Desta forma o presente estudo registra pela primeira vez um ginandromorfo de A. dubitatum, ampliando assim os relatos de carrapatos ginandromorfos no território brasileiro.

No presente trabalho, foram ainda encontradas as espécies $A$. sculptum e A. aureolatum que são consideradas importantes vetoras da bactéria Rickettsia rickettsii agente etiológico da febre maculosa brasileira, tais encontros corroboram com outras pesquisas recentes desenvolvidas em Zoológicos no estado de São Paulo (MARTINS et al., 2015; GONZALEZ et al., 2017; TEIXEIRA et al., 2017). 

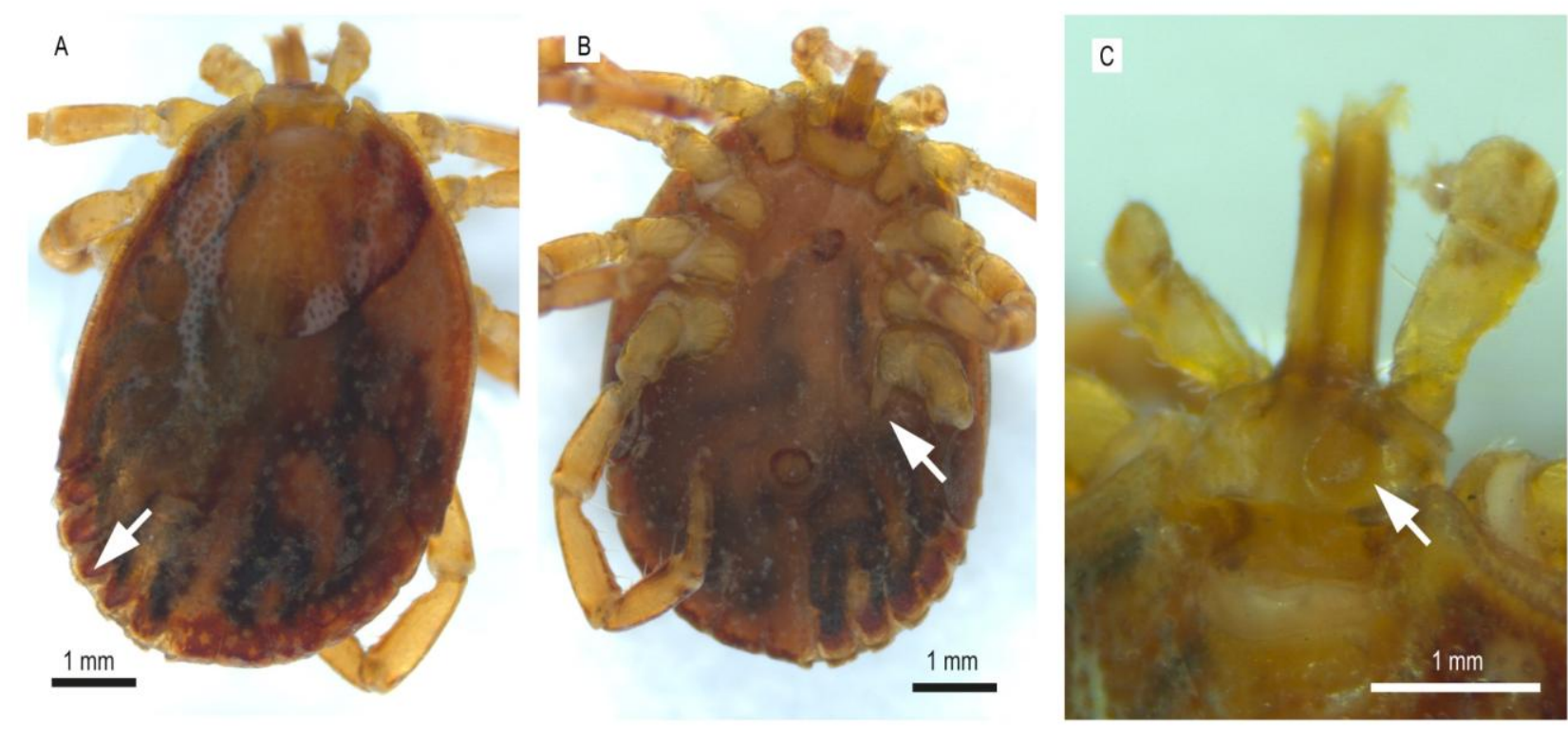

Figura 1 - Ginandromorfo de Amblyomma dubitatum coletado em Hydrochoerus hydrochaeris no Zoológico Municipal de Guarulhos, estado de São Paulo, Brasil. A e C: vista dorsal; Em destaque, os festões com prolongamentos quitinosos moderados do lado masculino e a área porosa na base dorsal do capítulo do lado feminino. B: vista ventral; Em destaque, o espinho longo na coxa IV do lado masculino.

\section{CONCLUSÃO}

A inspeção de animais silvestres de cativeiro e principalmente dos animais de vida livre recémchegados da natureza em Jardins Zoológicos, são importantes fontes para a detecção de ectoparasitas. A coleta manual de carrapatos em animais durante os exames clínicos de rotina ou através da vigilância epidemiológica, assim como o tombamento de artrópodes coletados em Parques Zoológicos em coleções acarológicas de referência, assegura uma rica fonte de informações. Desta forma, podem ser úteis em futuras investigações, identificações e erradicações de ácaros exóticos em coleções de animais cativos de Zoológicos.

\section{AGRADECIMENTOS}

O presente trabalho recebeu auxílio financeiro da Fundação de Amparo à Pesquisa do Estado de São Paulo (FAPESP), do Conselho Nacional de Desenvolvimento Científico e Tecnológico (CNPq) e da Coordenação de Aperfeiçoamento de Pessoal de Nível Superior (CAPES).

\section{REFERÊNCIAS}

ADLER, P. H.; TUTEN, H. C.; NELDER, M. P. Arthropods of Medicoveterinary Importance in Zoos. Annual Review of Entomology, v.56, p.123-142, 2011.

ALMEIDA, A. F.; VASCONCELLOS, M. K. Fauna Silvestre: Quem são e onde vivem os animais na metrópole paulistana. São Paulo: Secretaria Municipal do Verde e do Meio Ambiente, 2007. 350 p.
BARROS-BATTESTI, D. M.; ARZUA, M.; BECHARA, G. H. Carrapatos de importância médico-veterinária da região neotropical: um guia ilustrado para identificação de espécies. São Paulo: Vox/ICTTD3/Butantan, 2006. 223 p.

FONSECA, F. Notas de Acareologia. XXI. Gymnandromorphismo em Amblyomma cajennense (Fabr., 1787). Memórias do Instituto Butantan, v.10, p.39-41, 1935.

GONZALEZ, I. H. L.; LABRUNA, M. B.; CHAGAS, C. R. F.; SALGADO, P. A. B.; MONTICELLI, C.; MORAIS, L. H.; MORAES, A. A.; ANTUNES, T. C.; RAMOS, P. L.; MARTINS, T. F. Ticks infesting captive and free-roaming wild animal species at the São Paulo Zoo, São Paulo, Brazil. Revista Brasileira de Parasitologia Veterinária, 2017, Aceito.

GUGLIELMONE, A. A.; ESTRADA-PEÑA, A.; KEIRANS, J. E.; ROBBINS, R. G. Ticks (Acari: Ixodida) of the Neotropical Zoogeographic Region. Atalanta, Houten, The Netherlands, International Consortium on Ticks and Tickborne Diseases (ICTTD-2), 2003. 173 p.

IBGE Instituto Brasileiro de Geografia e Estatística. Brasil: Censo Demográfico, 2016. Disponível em: <http://www.ibge.gov.br/home $>$. Acessado em: 22 fev. 2017.

LABRUNA, M. B.; HOMEM, V. S. F., HEINEMANN, M. B.; FERREIRA NETO J. S. A case of gynandromorphism in Amblyomma oblongoguttatum (Acari: Ixodidae). Journal of Medical Entomology, v.37, p.777-779, 2000. 
LABRUNA, M. B.; JORGE, R. S. P.; SANA, D. A.; JACOMO, A. T. A.; KASHIVAKURA, C. K.; FURTADO, M. M.; FERRO, C.; PEREZ, S. A.; SILVEIRA, L.; SANTOS, T. S.; MARQUES, S. R.; MORATO, R. G.; NAVA, A.; ADANIA, C. H.; TEIXEIRA, R. H. F.; GOMES, A. A. B.; CONFORTI, V. A.; AZEVEDO, F. C. C.; PRADA, C. S.; SILVA, J. C. R.; BATISTA, A. F.; MARVULO, M. F. V.; MORATO, R. L. G.; ALHO, C. J. R.; PINTER, A.; FERREIRA, P. M.; FERREIRA, F.; BARROS-BATTESTI, D. M. Ticks (Acari: Ixodida) on wild carnivores in Brazil. Experimental and Applied Acarology, v.36, p.149-163, 2005.

LABRUNA, M. B.; ONOFRIO, V. C.; BEATI, L.; ARZUA, M.; BERTOLA, P. B.; RIBEIRO, A. F.; BARROS-BATTESTI, D. M. Redescription of the female, description of the male, and several new records of Amblyomma parkeri (Acari: Ixodidae), a South American tick species. Experimental and Applied Acarology, v.49, p.243-260, 2009.

LABRUNA, M. B.; PINTER, A.; TEIXEIRA, R. H. F. Life cycle of Amblyomma cooperi (Acari: Ixodidae) using capybaras (Hydrochaeris hydrochaeris) as hosts. Experimental and Applied Acarology, v.32, p.79-88, 2004.

LABRUNA, M. B.; RIBEIRO, A. F.; CRUZ, M. V.; CAMARGO, L. M; CAMARGO, E. P. Gynandromorphism in Amblyomma cajennense and Rhipicephalus sanguineus (Acari: Ixodidae). Journal of Parasitology, v.88, p.810-811, 2002.

LABRUNA, M. B.; SANFILIPPO, L. F.; DEMETRIO, C.; MENEZES, A. C.; PINTER, A.; GUGLIELMONE, A. A.; SILVEIRA, L. F. Ticks collected on birds in the state of São Paulo, Brazil. Experimental and Applied Acarology, v.43, p.147-160, 2007.

LUZ, H. R.; FACCINI, J. L.; LANDULFO, G. A.; COSTA NETO, S. F.; FAMADAS, K. M. New records for Amblyomma sculptum (Ixodidae) on non-passerine birds in Brazil. Revista Brasileira de Parasitologia Veterinária, v.25, p.124-126, 2016.

MARTINS, T. F.; BARBIERI, A. R.; COSTA, F. B.; TERASSINI, F. A.; CAMARGO, L. M.; PETERKA, C. R.; PACHECO, R. C.; DIAS, R. A.; NUNES, P. H.; MARCILI, A.; SCOFIELD, A.; CAMPOS, A. K.; HORTA, M. C.; GUILLOUX, A. G.; BENATTI, H. R.; RAMIREZ, D. G.; BARROS-BATTESTI, D. M.; LABRUNA, M. B. Geographical distribution of Amblyomma cajennense (sensu lato) ticks (Parasitiformes: Ixodidae) in Brazil, with description of the nymph of $A$. cajennense (sensu stricto). Parasites \& Vectors, v.9, p.186, 2016.

MARTINS, T. F.; MILANELO, L.; KRAWCZAK, F. S.; FURUYA, H. R.; FITORRA, L. S.; DAS DORES, F. T.; PEDRO, V. S.; HIPPOLITO, A. G.; LABRUNA, M. B. Diversity of ticks in the wildlife screening center of São Paulo city, Brazil. Ciência Rural, v.47, p.1-6, 2017,
MARTINS, T. F.; ONOFRIO, V. C.; BARROSBATTESTI, D. M.; LABRUNA, M. B. Nymphs of the genus Amblyomma (Acari: Ixodidae) of Brazil: descriptions, redescriptions, and identification key. Ticks and Tick-borne Diseases, v.1, p.75-99, 2010.

MARTINS, T. F.; TEIXEIRA, R. H. F.; LABRUNA, M. B. Ocorrência de carrapatos em animais silvestres recebidos e atendidos pelo Parque Zoológico Municipal Quinzinho de Barros, Sorocaba, São Paulo, Brasil. Brazilian Journal of Veterinary Research and Animal Science, v.52, p.319-324, 2015.

OGRZEWALSKA, M.; SARAIVA, D. G.; MORAESFILHO, J.; MARTINS, T. F.; COSTA, F. B.; PINTER, A.; LABRUNA, M. B. Epidemiology of Brazilian spotted fever in the Atlantic Forest, state of São Paulo, Brazil. Parasitology, v.139, p.1283-1300, 2012.

OLIVEIRA, G. M. B.; MARTINS, T. F.; MACHADO, L. C. P.; NICOLA, P. A.; HORTA, M. C. Ocorrência de carrapatos em Tamandua tetradactyla (Linnaeus, 1758) na Caatinga, nordeste do Brasil. Arquivo Brasileiro de Medicina Veterinária e Zootecnia, v.69, p.865-869, 2017.

PEREIRA, C.; CASTRO, M. P. Sobre um ginandromorfo de Rhipicephalus sanguineus Latr., 1804. Arquivos do Instituto Biológico, v.10, p.187-192, 1945.

PIEDADE, H. M. Fauna Urbana. São Paulo: Secretaria do Meio Ambiente/Coordenadoria de Educação Ambiental/Cadernos de Educação Ambiental, 17, v.2, 2013. 176 p.

SÁNCHEZ-CORDERO, V.; MEDELLÍN, R. A. Contribuciones mastozoológicas en homenaje a Bernardo Villa. Instituto de Biología-UNAM, Instituto de Ecología-UNAM y Comisión Nacional para el Conocimiento y Uso de la Biodiversidad (Conabio), México, D.F., 2005. 679 p.

TEIXEIRA, R. H. F.; LABRUNA, M. B.; MARTINS, T. F. Ixodídeos coletados parasitando animais selvagens no Zoológico de Sorocaba, estado de São Paulo, Brasil. Revista de Educação Continuada em Medicina Veterinária e Zootecnia do CRMV-SP, v.15, p.8-14, 2017.

WITTER, R.; MARTINS, T. F.; CAMPOS A. K.; MELO, A. L. T.; CORRÊA, S. H. R.; MORGADO, T. O.; WOLF, R. W.; MAY-JÚNIOR, J. A.; SINKOC, A. L.; STRÜSSMANN, C.; AGUIAR, D. M.; ROSSI, R. V.; SEMEDO, T. B. F.; CAMPOS, Z.; DESBIEZ, A. L. J.; LABRUNA, M. B.; PACHECO, R. C. Rickettsial infection in ticks (Acari: Ixodidae) of wild animals in midwestern Brazil. Ticks and Tick-borne Diseases, v.7, p.415-423, 2016. 